\title{
Commuters' Willingness to Shift to Metro: a Case Study of Salaya, Thailand
}

\author{
Anna Fraszczyk $^{1}$ (1) $\cdot$ Waressara Weerawat $^{1} \cdot$ Phumin Kirawanich $^{1}$
}

Received: 19 June 2019/Revised: 17 September 2019/Accepted: 9 October 2019/Published online: 4 November 2019

(C) The Author(s) 2019

\begin{abstract}
The rapid expansion of new metro systems in the last two decades shows a globally growing interest in urban rail transit infrastructure. Every time a new metro line is opened, it has the potential to change the existing modal split, which is usually dominated by private (car or motorcycle) transport users. For each new or expanding metro system, a feasibility study is conducted, which focusses mainly on existing mobility issues, travel demand, new infrastructure proposed, and environmental and financial aspects of a new metro project. In these documents, specific methodologies are applied, using local parameters and suitable software, for demand forecasting purposes. However, not much attention is usually dedicated to potential users, their modal choice characteristics, willingness to shift to metro and conditions under which such a shift would be encouraging. Therefore, it is often unclear what the actual public perception of a new service is and their interest in using it in the future. As a consequence of various shortcomings in feasibility studies, the actual demand for metro services is often much lower than originally predicted. Hence, a survey targeting a group of potential metro users located close to a planned metro line was designed to investigate people's willingness to shift to metro and include soft factors that could facilitate such a change. Next, a case study of the Red Line whose final metro stop is at Salaya, Thailand, was selected, and a sample of 667 respondents, both staff and students based at
\end{abstract}

Anna Fraszczyk

anna.fra@mahidol.edu; anna.fraszczyk@gmail.com

1 Cluster of Logistics and Rail Engineering, Faculty of Engineering, Mahidol University, 25/25 Phuttamonthon 4 Road, Salaya, Nakhon Pathom 73170, Thailand

Communicated by Marin Marinov. a nearby university campus, was investigated. Results revealed that $90 \%$ of the sample is willing to shift to metro (easy and medium shifters) for a future 20-km trip between Salaya and Bangkok, while only $10 \%$ remains sceptic (difficult shifters). Moreover, there are some differences in shifters' priorities, where easy shifters see factors such as reliable timetable, good mode connectivity and seamless station-campus connection as significantly more important than medium or difficult shifters do. Also, there is a significant association between a mode type currently used for Salaya and Salaya-Bangkok commutes, where private transport users (51\% of staff and $43 \%$ of students) have already shifted to public transport. Based on the outcomes of the study, recommendations are addressed to stakeholders involved in the Red Line, as well as in other new metro projects in Thailand and internationally, and to those who aim to better understand the needs of potential customers in order to facilitate their shift to metro as a preferred mode of transport in the future.

Keywords Mode shift · Commute mode · Metro · Survey · Salaya

\section{Introduction}

The global number of metro systems is currently growing, with over 70 new metro systems opened in the last two decades [1], mainly in developing countries. This is followed by increased interest in a wide range of metro research and reports, from feasibility studies to operation enhancement or passenger satisfaction surveys, which are conducted to better understand the need for these systems and how to improve their operations, from both technical and passengers' perspectives. However, research focussed 
Table 1 Examples of barriers to modal shift (from private to public transport) Source: Derek Halden Consultancy [5]

\begin{tabular}{lll}
\hline Hard barriers & Soft barriers & Complementary barriers \\
\hline Travel time & Information & Non-transport costs and taxes \\
Value of time & Flexibility (tickets) & Limited travel time budgets \\
Travel cost & Comfort & Need to carry goods \\
Reliability of PT & Security & Workplace constraints \\
Capacity & Personal and social needs and attitudes & Lifestyle \\
Limited coverage (no door-to-door service) & & Weather \\
Car ownership levels & & Societal trends \\
Land use & & \\
\hline
\end{tabular}

on shift mechanisms from other transport modes to metro is a relatively new area. Some recent examples include work on people's pre-launch attitudes to new metro systems, which aimed to understand public perception of a new driverless train service [2] or competitiveness of a new metro line when compared with other modes of transport available [3].

This paper is one of the few looking at people's willingness to shift from their current modes of transport to metro, conducting a survey prior to the new service launch. It is believed that new knowledge gained from this approach will allow metro operators and authorities to access new information, which could help them understand potential users' perspectives better and facilitate new decisions to meet their expectations regarding the new service.

A survey was conducted to assess people's willingness to shift from their current modes of transport to metro in the future and conditions under which such a shift would be considered. A case study was selected along one of the new metro lines in Bangkok, Thailand. Based on the results of the analysis, a set of recommendations for the metro stakeholders on how to manage respondents' willingness to shift to metro and preferred conditions which would accommodate such a shift are listed. This study could also serve as a lesson for other new metro systems, which aim to understand better the shift mechanism to metro from their potential customers' perspective in order to meet their expectations after the new metro line is launched.

The paper is organised in six sections. First, the topic of the study is introduced. Second, the context of the paper is explained with a review of the literature on modal choice, metro feasibility studies and shift to metro. Based on the literature review, the research gap is introduced, and the case study is explained. Third, the methodology applied in the study is described. Fourth, the results of the survey are presented and discussed. Next, conclusions and recommendations are listed, before the final section explains the study limitations and suggests next steps.

\section{Background}

\subsection{Modal Choice}

Usually a trip starts with a motive. Four travel motives have been identified: home-work commuting, homeschool commuting, business and leisure [4]. The way people choose to travel to their destinations is based on a complex set of factors and, as stated in Ref. [4], this can take place consciously or unconsciously. This process is called modal choice, and three approaches to it can be distinguished: rationalist (where time and cost play a key role), socio-geographical (with spatial component) and socio-psychological (with attitudes), each with their own characteristics. The rationalist approach is most popular in the literature, and it is based on maximising time and cost parameters [4]. There are over 20 different modal choice determinants, some of which are studied more often (e.g. car availability, age, gender, income) than others (e.g. trip chaining, lifestyle, perceptions, habits) [4].

Barriers and bridges to modal shift were investigated in Ref. [5]. The authors split barriers into three categories: hard, soft and complementary. Examples of such barriers, in the context of a shift from private to public transport (especially rail), are listed in Table 1.

Soft barriers to public transport are related to the way it is perceived by people and the quality of the experience it offers. It has been observed that more countries, especially developed countries, are focussing more on sustainable transport options, including public transport, which brings benefits of an environmental and economical nature, and this approach has been enforced by the United Nations in their ongoing Sustainable Development Goals strategy [6].

Derek Halden Consultancy [5] points out that there may be multiple barriers to modal shift at a time and "tackling one or two issues will have no impact if other barriers remain" as changes in travel behaviour will only occur when "all the relevant barriers have been addressed" ( $p$. 63). However, bridges to modal shift have also been 
recognised, and these can be divided into four categories: improvements to alternatives, making car travel less attractive, management and administration, and technology information and marketing. The longest list of bridges could be identified as various types of improvements that could be made by local authorities and public transport operators, and related to [5]:

- The quality of the waiting environment (bus stops and rail stations)

- Improved security (via CCTV and reduced vandalism)

- Electronic and printed information

- Larger station car parks

- Enforcement of bus lanes

- More priority to pedestrians (e.g. safe pavements)

- Better multimodal integration (interchanges, timetables, information and ticketing)

In addition, highlighting benefits of public transport, from better environment for all to improved well-being of individuals, could help people to relate their mode shift to greater positive changes in their local communities.

\subsubsection{Modal Choice Modelling}

The purpose of modal choice modelling is to analyse the choices that people (individuals or groups) make in selecting transport modes, and these choices can be different for different types of trip [7]. Therefore, the objective of such modelling is to predict the share of trips made by mode, and often the share of trips by public transport [7].

Techniques used in mode choice modelling have evolved over the decades, and in general, two types of techniques can be distinguished: probabilistic techniques using statistics and modern artificial intelligence-based techniques using computational intelligence (CI). Probabilistic techniques belong to a more traditional approach, with probit or logit models popular in the 1960s and 1970s and the nested logit model popular since the 1990s till the present, while modern artificial intelligence-based techniques have been applied since 1990s and include artificial neural networks (ANNs) and fuzzy logic systems [8]. A detailed review of various techniques is offered in Ref. [8], where the authors go into details of each approach (with their advantages and disadvantages), introduce equations and provide specific examples that have been applied in the transport literature over the last few decades. Developing and implementing realistic models to study modal choice is important, as it can affect the evaluation of new transport options.

\subsection{Metro Feasibility Studies}

Most planned new or extension metro lines will have a feasibility study done first to assess the need for and practicality of the solutions proposed. A feasibility study is a report focussed mainly on existing or predicted mobility problems (e.g. congestion, reliability and crowding) and how a new metro (extension, line or network) could help to solve them. These documents often include sections on (existing and future) travel demand, infrastructure design, environmental and socio-economic impacts, and financial commitments needed [9-12]. In addition, some feasibility studies might include a few different route scenarios [12], traffic volume calculation methods [9], emissions reduction [9] or comparisons with other similar systems [10]. Also, issues such as metro stations' interchange access [13], sustainability issues [14] or passenger satisfaction surveys [10] are sometimes targeted by additional and detailed metro reports, some of which show that, from the metro/ rail passengers' perspective, punctuality and reliability are key factors influencing their satisfaction [15].

Overall, although being very informative about technical aspects of new metro projects, what these feasibility studies and reports often fail to do is to present soft measures and evidence of potential mode shift. A set of soft and cultural factors surrounding mode shift, such as perceptions of security, social needs, personal attitudes or society lifestyle, are often neglected in feasibility studies. Outcomes of potential users' surveys, with their attitudes towards a new metro, willingness (or lack thereof) to shift to a new mode and conditions under which such a shift would be considered, if at all, could bring a new perspective to new metro project planning. This aspect is frequently ignored in feasibility studies or other pre-launch metro reports, as it is simply assumed that people will use the new metro service (shift to metro) as soon as it is available. However, some examples show that this mode shift process is not always as smooth as expected.

It has indeed been found that various metro systems around the world experienced much lower demand for metro projects in the opening year than forecasted (e.g. Calcutta metro 5\%, Miami metro $15 \%$, Tyne and Wear Metro 50\%) [16], and these trends often continue in later operations. Evidence shows that, in many cases, metro ridership forecasts were inaccurate and overestimated by $65 \%$ on average, and these numbers are usually much higher for rail than for road megaprojects [17]. Two striking examples are the Calcutta metro, with ridership numbers just above $10 \%$ of forecasts, and the Bangkok Skytrain, with overestimated passenger forecasts as well as station and platform dimensions, at least for their early operations [17]. 
Reasons for demand prediction failures are often multiple, but Ref. [18] distinguished three types related to: technical (e.g. unreliable data used for analyses), psychological and political-economic (e.g. appraisal bias of the project) promoter issues, each with its own characteristics. Reference [19] gives specific examples of parameters that were wrongly assumed in a feasibility study for the Calcutta metro and influenced the demand calculation, which was eventually wrong. These parameters, assumed at the planning stage, were related to [19]:

- Much higher growth rates (population and economic) than actual

- Operation of a larger metro network (two lines) in the future

- Shorter than actual headways and inaccurate traffic modelling

- Feeder bus services serving metro stations

In addition, an issue of bus competition occurred, which offered metro line routes, lower prices and door-to-door service [19].

Similar examples of incorrect demand forecasting can be found in Bangkok. The new Purple Line in Bangkok, launched in 2017, in its first year after the launch achieved a much lower patronage of about 22,000 passengers per day than the 100,000 originally predicted [20]. This was mainly due to a 'missing link' issue, which failed to offer passengers seamless connection between the new Purple Line and the existing Blue Line [21], and it took 1 year to fix this. Although the numbers increased to about 50,000 once the seamless connection between the two metro lines was established, the current patronage is still about half of what was expected [22, 23].

Another example from the Sydney Metro shows that their new driverless metro system, whose phase one was launched in May 2019, experienced daily patronage of 72,000 in its first week of operation, which is seen as a success [24]. However, the overall system's capacity is targeted to be much greater, about 40,000 passengers per hour [25]. If this target is to be achieved, after all phases are completed, it may be necessary to obtain a deeper understanding of potential passengers' needs and their hopes from the new system and act upon it to achieve the expected scale of mode shift.

Overall, the examples presented above show that simply opening a new metro line to the public is not enough reason for them to use it in the quantities forecasted. The evidence shows that what is predicted to happen, in terms of demand forecasting, might not necessarily happen due to various reasons, including incorrect data assumptions, people's embedded perceptions, travel habits or lifestyle. Therefore, metro stakeholders should be aware that any forecasted change in travel behaviour, and people's shift from their previous modes to metro, might take longer than expected and that the scale of the shift might differ from the scale assumed in feasibility reports.

\subsection{Shift to Metro Research}

There is a limited number of publications focussed on passengers' shift to metro, despite the fact that metro systems are expanding globally and offering an effective tool to fight congestion and pollution [11]. When looking in detail, most new systems are being built in developing countries [1], where often infrastructure construction comes first and travel behaviour research next, if at all.

People's pre-launch attitudes to metro services have been investigated previously at a small scale in both developed [2] and developing [3, 26] countries. Fraszczyk and Mulley [2] focussed on new driverless metro trains in Sydney and people's perceptions of this new mode of transport which does not require a driver. It was found that the majority of their 300 respondents were positive about the new driverless metro service, but still they expected to see some traditional features on a train (e.g. driver's cabin) whose inclusion is no longer technically justified on this type of service. It was recommended to put more effort into new service marketing actions targeting various types of potential customers so that they understand features of the new driverless system better and are comfortable using it in the future.

Sohoni et al. [26] investigated the mode shift behaviour of commuters in Mumbai by conducting two surveys: a revealed preference (RP) survey (actual behaviour), with a sample of 153, and a stated preference (SP) survey (hypothetical scenarios), with a sample of 169 respondents. Results of the RP survey show that a great majority of respondents (approx. 80\%) were already public transport users before they shifted to a new metro line. Also, over half of the respondents (approx. 60\%) in the SP survey who used a private vehicle showed willingness to shift to a proposed metro line [26]. The authors also claim that results of their analysis were fed into transport planning and modelling activities related to ridership estimation and value of travel time savings on a proposed new metro corridor.

Dahlan et al. [3] examined people's pre-launch perceptions of a new metro service in Jakarta, which was to be the first metro system in Indonesia. They classified the 516 respondents into two groups: those located along the new metro corridor and those located beyond the metro corridor in other areas of the city, and identified few significant differences between the two. The metro corridor group was less likely to own a private vehicle and more interested in choosing the metro option as an alternative to other transport modes than the other group. These findings could 
inform the new metro operator and other stakeholders about people's expectations from the new service. Moreover, service priority parameters (time, cost, effort and $\mathrm{CO}_{2}$ emissions) and other key factors, such as reliability and ticket price, were identified for the majority of the sample, as they could influence future metro patronage.

\subsection{Research Gap}

The literature focussed on new metro lines often deals with technical issues of infrastructure design or operations, but lacks an insight from potential passengers' point of view. There is very limited research on people's pre-launch willingness to swap their current modes of transport in favour of the new metro. Therefore, this paper aims to look into people's willingness to shift to metro and conditions under which they would be interested in doing so. A case study approach based on a developing country is applied to inform the new metro stakeholders and decision-makers about potential users' attitudes towards the new system and the importance of factors, both hard and soft, that could facilitate the future shift to metro.

\subsection{Salaya Case Study}

Salaya is a sub-district located $20 \mathrm{~km}$ west of Bangkok, Thailand, and is expected to have a direct metro connection (Red Line) with the capital by 2022. The Red Line development is part of a larger metro expansion project called the Mass Rapid Transit Master Plan in Bangkok Metropolitan Region [27, 28], which aims to increase the total length of the metro network in Bangkok from the existing $100 \mathrm{~km}$ (in 2019) to $500 \mathrm{~km}$ in the future. This new line is unique in a way as its tracks will be built on the existing railway route from Bang Sue to Salaya Station, which is located approximately $150 \mathrm{~m}$ from a large university campus. Currently, the railway services in Thailand, including the operation between Salaya and Bangkok, are underinvested and underused, and require urgent reforms [29]. Moreover, construction works on the Red Line have been delayed for years, and it is not clear what the actual willingness of potential commuters to shift from their current modes of transport to metro would be. Therefore, a sample of potential commuters based at Salaya Campus, who also commute between Salaya and Bangkok, have been invited to take part in the study and share their willingness to shift to metro and elaborate on conditions under which such a shift would be possible. The selected sample is unique in at least two ways: the university sample is atypical in its demographics (e.g. large proportion of young respondents), and the close location of the campus to the new Salaya Station is atypical spatially.

\section{Methodology}

\subsection{Questionnaire Design}

The study used a quantitative approach with a questionnaire as a tool for data collection. The questionnaire designed for the study was inspired by various university travel surveys conducted on a regular basis in Australia [30], the USA [31] and the UK [32, 33]. It was divided into three key parts, and the main focus was to collect data about (1) staff and students currently commuting to/from the campus, as well as (2) their willingness to shift to a new metro line when commuting between Salaya and Bangkok in the future.

Part one of the questionnaire looked at daily travel behaviour and included questions related to the characteristics of respondents' commuting:

- Commuting distance (within $2 \mathrm{~km}$, between 2 and $5 \mathrm{~km}$, over $5 \mathrm{~km}$, or accommodation on the campus)

- Frequency of commuting* (from less than once a week to 7 days per week, or other)

- Usual mode of commuting to/from and travel on the campus* (15 different mode options, or other)

- Commuting alternatives and reasons why they are not being used (15 different mode options, or other)

- Travel time (in minutes), leave time (specific time) and cost (in Thai baht)

- Usual mode of Salaya-Bangkok commuting* (15 different mode options, or other)

Part two focussed on respondents' opinions and preferences regarding the new metro service and asked about:

- Importance of ten variables when choosing a commute mode (e.g. low price, good for the environment)

- Ideal one-way commute time

- Future preference for metro as an option for SalayaBangkok commute mode*

- Importance of ten variables when deciding on metro option for Salaya-Bangkok commute*

- Importance of three parameters when choosing a feeder option between the campus and the metro station* (short time, low cost, minimum effort)

- Suggestions for promotion of transport options and 'green campus' approach (e.g. invest in cycling, promote public transport, and other)

More specifically, the key question in this section in the context of the analysis presented later in the paper asked: "If in the future you have a return business/university meeting in Bangkok city centre during work/study hours and you start and finish your journey at [...] Salaya campus, would you consider the new Red Line as an option?"

Four answer options were available: 
- 'Yes, I could use Red Line every time';

- 'Yes, I could use Red Line sometimes';

- 'No' (please explain why);

- 'Other, please specify'.

Part three looked at respondents' socio-economic data and asked 11 questions related to: gender* (male, female or prefer not to say), age*, employment status* (nine options, from undergraduate year 1 to exchange staff), faculty, mobility tools (e.g. driver's licence, access to a private bicycle), number of people and vehicles in household, home address, date since at the current address, date since at this university and disabilities. Part four offered an open space for additional comments related to the survey.

The survey was designed in an online and an offline (paper) mode. Results presented in this paper focus on the responses given to the total of nine questions, marked with a star $(*)$ above (9 out of 28 ), which are more relevant to the shift to metro topic.

\subsection{Data Collection}

The survey was launched on 5 May 2017, and data collection (online and offline) continued until 20 June 2017. The survey targeted staff and students commuting to or living on the Salaya University Campus only (the university has five other campuses in Thailand, and some staff and students travel between campuses). The total number of responses collected was 742 , but after a data cleaning process was employed, respondents who commute less than once a week $(n=71)$ or did not specify commuting patterns $(n=4)$ were excluded. The final sample size used in the analysis included 667 respondents. Next, IBM SPSS and MS Excel were employed to analyse the results.

\section{Analysis of Results}

\subsection{Sample Size and Socio-economics}

The sample size included in the study was 667 respondents with a split of 390 staff (58\%) and 277 students (42\%). The staff group was older than the student group, with over half of staff respondents being between 31 and 45 years of age. Among the students, $42 \%$ were aged between 17 and 20 years, followed by $34 \%$ between 21 and 25 years. Details are displayed in Fig. 1. In addition, the gender split in the sample was $60 \%$ female and $39 \%$ male, with $1 \%$ preferring not to say.

The results show that $70 \%$ of staff respondents commute to Salaya campus 5 days a week in comparison with only $39 \%$ of students. Also, $17 \%$ of students but only $4 \%$ of staff respondents live on the campus, as shown in Fig. 2. Two

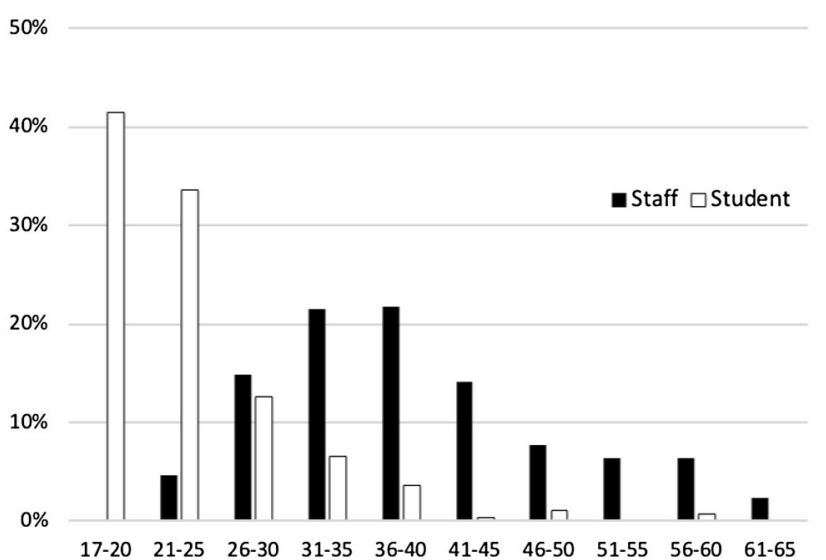

Fig. 1 Respondents' age (\%)

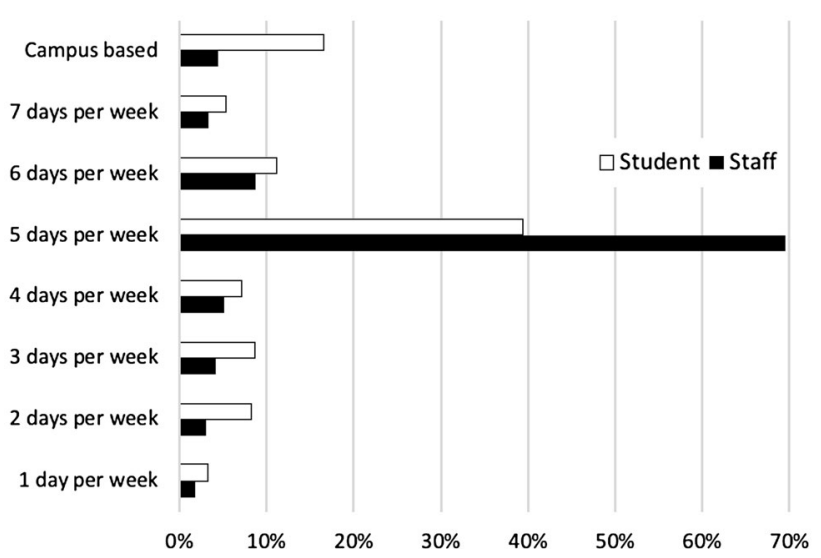

Fig. 2 Respondents' commute frequency (\%)

key transport modes used by $80 \%$ of all respondents are either in a form of private transport, which includes driving, being a passenger in a car or driving a motorcycle; or public transport, which includes a bus, shuttle bus, metro (BTS - Bangkok Mass Transit System, MRT - Metropolitan Rapid Transit or ARL - Airport Rail Link) or train mode. Private transport as a primary mode of commute is used by $56 \%$ of staff and $25 \%$ of students, while public transport is used by more students (45\%) than staff (32\%), as seen in Fig. 3.

In terms of Salaya versus Salaya-Bangkok commute trips, the results displayed in Table 2 show that there is a significant association between the transport modes used on the two routes (chi-squared test; $p<0.001$ ). About $30 \%$ of those who commute to Salaya using private transport will continue to do so for their Salaya-Bangkok trips, but half of the staff who commute to Salaya using private transport would shift to public options for their Bangkok commute (50.9\%), compared with only $42.6 \%$ of students. An overwhelming majority of staff and students who already commute to Salaya by public transport report that they also use this form of transport to get to Bangkok (83.9\% staff 


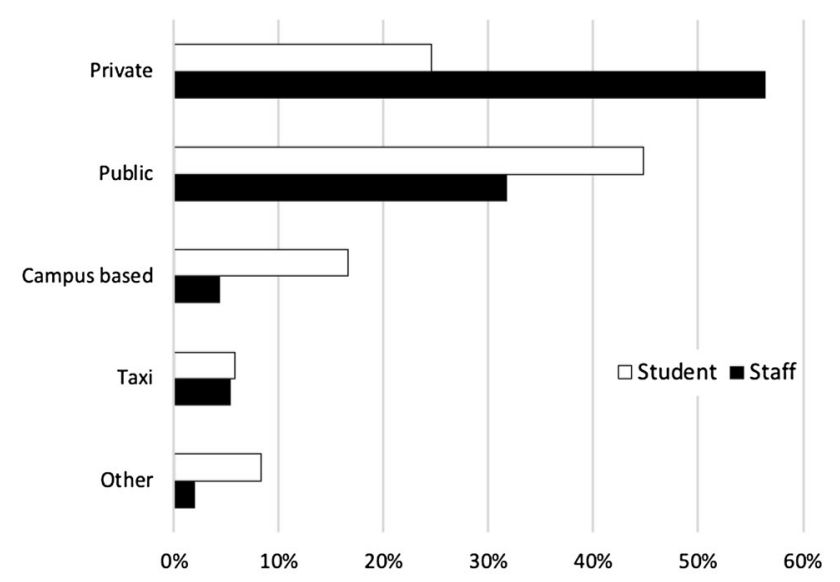

Fig. 3 Respondents' primary commute mode (\%)

and $79.8 \%$ students). These results show that the shift from private to public transport is already happening, although the scale of this behaviour is different amongst staff and students. Specific factors that could potentially facilitate the shift to metro are investigated in Sect. 4.3.

\subsection{Willingness to Shift to Metro}

The respondents were asked about their willingness to shift from their current modes to the new metro line in the future when commuting between Salaya and Bangkok (Sect. 3.1). The four answer options were grouped into three types of shifters:

- Easy shifters, who replied: 'Yes, I could use Red Line every time'

- Medium shifters, who replied: 'Yes, I could use Red Line sometimes'

- Difficult shifters, who replied: 'No' or 'Other, please specify'

The results displayed in Table 3 show that the combined numbers for easy shifters (260 respondents) and medium shifters (365 respondents) represented over $90 \%$ of the sample. In these two categories, the split between staff and students is about $6: 4$, but within the difficult shifters type (42 respondents only) the proportion changes to about 7:3, meaning that the share of staff in this category is greater than in the former two. Results of chi-squared test show no statistically significant differences between respondents' shifters' categories and their status, gender or SalayaBangkok commute mode.

Table 3 displays selected characteristics of three types of metro shifters. In terms of gender, a great majority in each of the shifters' types are female, but they also form $60 \%$ of the overall sample. However, in the medium shifters category the split is even greater with $64 \%$ of females but only $35 \%$ of males. The three types of shifters have quite similar splits of transport modes currently used for Salaya-Bangkok commuting, with the most popular being public transport (between 64\% and 69\%) and private transport options coming next (between $14 \%$ and 16\%) with the exception of the difficult shifters type, among whom 'Other' category respondents represent $17 \%$.

As the gender split within staff and students groups is similar, respondents' age within each type of the shifters was analysed, as seen in Fig. 1. The first two age groups (age 17-25 years) are dominated by students, the 26-30year age category has a fairly equal split between staff and students, and the remaining categories are dominated by staff. Figure 4 shows that, overall, the older the respondents are the greater the proportion of easy shifters to medium shifters is, starting with $39 \%$ versus $57 \%$ for the $17-20$-year-old category and ending with $55 \%$ versus $44 \%$ for the 61-65-year-old respondents. However, there is an unexplained exception for the 51-55-year-old category, where only $12 \%$ are easy shifters while $64 \%$ are medium shifters. Also, the proportion of difficult shifters grows with age categories, from 3\% at the start point (and these are student respondents only) to $26 \%$ in the 56-60-year-old category (majority of staff respondents). This suggests that, overall for staff, the older they are, the more extreme their opinions become, either in favour (easy shifters) or against (difficult shifters) the shift to metro.

\subsection{Factors to Facilitate the Shift}

\subsubsection{Expectations from the Metro}

Next, the respondents were asked about factors that would encourage them to shift from their current transport modes to metro for their future Salaya-Bangkok commute trips. They rated 10 factors on a 5-point Likert scale, where 1 was 'not at all important', 3 was 'neutral', and 5 was 'very important'. The results were split for the three types of shifters and are displayed in Fig. 5 and Table 4.

Table 4 shows that the results for all but one factor (lower price) were significantly different between the shifters categories statistically. In general, the trends for all three types of shifters were similar within each factor's results, with five factors being ranked as 'very important' by a great majority within each group, viz. 'seamless station-campus connection', 'good mode connectivity', 'good safety', 'shorter trip time' and 'reliable timetable'. However, it must be highlighted that the easy shifters' numbers are higher (between $65 \%$ and $79 \%$ ) than those of the medium shifters (from 59\% to 65\%) or difficult shifters (from 59\% to 66\%) for these five key factors.

The second category of factors are those ranked as either 'important' or 'very important', where the combined proportion of respondents totals in the range of $70 \%$ for all 
Table 2 Respondents' primary transport mode for home-Salaya versus Salaya-Bangkok commute cross-tabulation

\begin{tabular}{|c|c|c|c|c|c|c|c|c|c|c|}
\hline & \multicolumn{10}{|c|}{ Primary Salaya-Bangkok commute mode } \\
\hline & \multicolumn{5}{|l|}{ Staff } & \multicolumn{5}{|l|}{ Students } \\
\hline & Private & Public & Other & No trips & Total & Private & Public & Other & No trips & Total \\
\hline \multicolumn{11}{|c|}{ Primary home-Salaya commute mode } \\
\hline \multicolumn{11}{|l|}{ Private } \\
\hline Count & 70 & 112 & 28 & 10 & 220 & 21 & 29 & 2 & 16 & 68 \\
\hline Expected count & 42.9 & 142.2 & 24.8 & 10.2 & 220 & 5.9 & 47.9 & 2.5 & 11.8 & 68 \\
\hline$\%$ within home-S mode & 31.8 & 50.9 & 12.7 & 4.5 & 100 & 30.9 & 42.6 & 2.9 & 23.5 & 100 \\
\hline$\%$ within S-BKK mode & 92.1 & 44.4 & 63.6 & 55.6 & 56.4 & 87.5 & 14.9 & 20.0 & 33.3 & 24.5 \\
\hline$\%$ of total & 17.9 & 28.7 & 7.2 & 2.6 & 56.4 & 7.6 & 10.5 & 0.7 & 5.8 & 24.5 \\
\hline Std. residual & 4.1 & -2.5 & 0.6 & 0 & & 6.2 & -2.7 & -0.3 & 1.2 & \\
\hline \multicolumn{11}{|l|}{ Public } \\
\hline Count & 4 & 104 & 10 & 6 & 124 & 1 & 99 & 5 & 19 & 124 \\
\hline Expected count & 24.2 & 80.1 & 14 & 5.7 & 124 & 10.7 & 87.3 & 4.5 & 21.5 & 124 \\
\hline$\%$ within home-S mode & 3.2 & 83.9 & 8.1 & 4.8 & 100 & 0.8 & 79.8 & 4.0 & 15.3 & 100 \\
\hline$\%$ within S-BKK mode & 5.3 & 41.3 & 22.7 & 33.3 & 31.8 & 4.2 & 50.8 & 50.0 & 39.6 & 44.8 \\
\hline$\%$ of total & 1.0 & 26.7 & 2.6 & 1.5 & 31.8 & 0.4 & 35.7 & 1.8 & 6.9 & 44.8 \\
\hline Std. residual & -4.1 & 2.7 & -1.1 & 0.1 & & -3 & 1.3 & 0.2 & -0.5 & \\
\hline \multicolumn{11}{|l|}{ Other } \\
\hline Count & 1 & 21 & 5 & 2 & 29 & 1 & 31 & 2 & 5 & 39 \\
\hline Expected count & 5.7 & 18.7 & 3.3 & 1.3 & 29 & 3.4 & 27.5 & 1.4 & 6.8 & 39 \\
\hline$\%$ within home-S mode & 3.4 & 72.4 & 17.2 & 6.9 & 100 & 2.6 & 79.5 & 5.1 & 12.8 & 100 \\
\hline$\%$ within S-BKK mode & 1.3 & 8.3 & 11.4 & 11.1 & 7.4 & 4.2 & 15.9 & 20.0 & 10.4 & 14.1 \\
\hline$\%$ of total & 0.3 & 5.4 & 1.3 & 0.5 & 7.4 & 0.4 & 11.2 & 0.7 & 1.8 & 14.1 \\
\hline Std. residual & -2 & 0.5 & 1 & 0.6 & & -1.3 & 0.7 & 0.5 & -0.7 & \\
\hline \multicolumn{11}{|l|}{ Campus based } \\
\hline Count & 1 & 15 & 1 & 0 & 17 & 1 & 36 & 1 & 8 & 46 \\
\hline Expected count & 3.3 & 11 & 1.9 & 0.8 & 17 & 4 & 32.4 & 1.7 & 8 & 46 \\
\hline$\%$ within home-S mode & 5.9 & 88.2 & 5.9 & 0.0 & 100 & 2.2 & 78.3 & 2.2 & 17.4 & 100 \\
\hline$\%$ within S-BKK mode & 1.3 & 6.0 & 2.3 & 0.0 & 4.4 & 4.2 & 18.5 & 10.0 & 16.7 & 16.6 \\
\hline$\%$ of total & 0.3 & 3.8 & 0.3 & 0.0 & 4.4 & 0.4 & 13.0 & 0.4 & 2.9 & 16.6 \\
\hline Std. residual & -1.3 & 1.2 & -0.7 & -0.9 & & -1.5 & 0.6 & -0.5 & 0 & \\
\hline \multicolumn{11}{|l|}{ Total } \\
\hline Count & 76 & 252 & 44 & 18 & 390 & 24 & 195 & 10 & 48 & 277 \\
\hline Expected count & 76 & 252 & 44 & 18 & 390 & 24 & 195 & 10 & 48 & 277 \\
\hline$\%$ within home-S mode & 19.5 & 64.6 & 11.3 & 4.6 & 100 & 8.7 & 70.4 & 3.6 & 17.3 & 100 \\
\hline$\%$ within S-BKK mode & 100 & 100 & 100 & 100 & 100 & 100 & 100 & 100 & 100 & 100 \\
\hline$\%$ of total & 19.5 & 64.6 & 11.3 & 4.6 & 100 & 8.7 & 70.4 & 3.6 & 17.3 & 100 \\
\hline
\end{tabular}

three categories of shifters. These factors are: 'good comfort', 'freedom of choice' and 'lower price'. Interestingly, the responses of all three types of shifters were most similar out of the ten factors for the 'lower price' factor, and they grow from an average of $4 \%$ for 'not at all important' to $19 \%$ for 'neutral' and $43 \%$ for 'very important'. Results for the 'good enjoyment' factor were the only ones to show a bell shape in Fig. 5, which means that, on average, $39 \%$ of respondents ranked it as a 'neutral' factor, with the remaining sample split between the "not important' and 'important' sides of the answer spectrum. Finally, just over $20 \%$ of respondents stated that the 'good for the environment' factor is 'neutral' to them (similar to the fraction of just under $20 \%$ for the 'lower price' factor), but the majority of the remaining shifters from each category agreed that this factor is either 'important' (30\% on average) or 'very important' ( $36 \%$ on average).

Table 4 sheds some more light on similarities and differences between the shifters types in terms of the means of the values for each of the ten factors. Overall, the 
Table 3 Selected characteristics of three types of metro shifters $(\%)$

\begin{tabular}{lcccr}
\hline & Easy shifters & Medium shifters & Difficult shifters & Total \\
\hline Status & & & & \\
Staff & 60 & 56 & 69 & 59 \\
Student & 40 & 44 & 31 & 42 \\
Total & 100 & 100 & 100 & 100 \\
Gender & & & & \\
Female & 54 & 64 & 57 & 60 \\
Male & 45 & 35 & 41 & 39 \\
Prefer not to say & 1 & 1 & 2 & 1 \\
Total & 100 & 100 & 100 & 100 \\
Salaya-Bangkok commute & mode & & & 15 \\
Private & 14 & 16 & 14 & 67 \\
Public & 69 & 66 & 64 & 4 \\
Taxi & 5 & 4 & 0 & 4 \\
Other & 4 & 3 & 17 & 10 \\
No trips & 10 & 11 & 5 & 100 \\
Total & 100 & 100 & 100 & 667 \\
Total (count) & 260 & 365 & 42 & \\
\hline
\end{tabular}

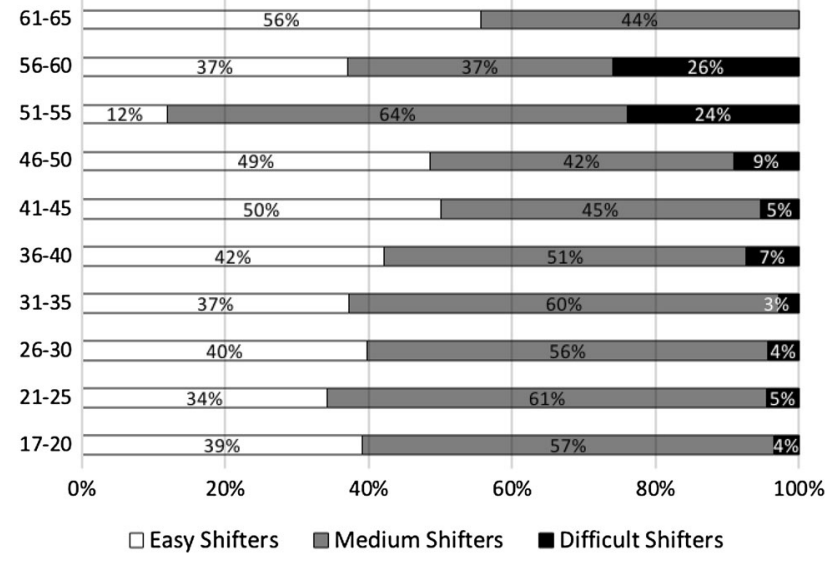

Fig. 4 Metro shifters' age categories (\%)

importance of the ten factors, from most to least important, is identical for the easy shifters and medium shifters, but slightly different for difficult shifters (e.g. the top factor is connectivity). The highest mean values are achieved for 'reliable timetable' factor, which scores 4.67 for easy and 4.43 for medium shifters types. The bottom factor shared across the three types of shifters is 'good enjoyment', whose achieved mean scores between 3.50 for easy shifters and 3.07 for difficult shifters. All three types of shifters agree that the top five factors are: 'reliable timetable', 'good mode connectivity', 'seamless station-campus connection', 'shorter trip time' and 'good safety', although for difficult shifters the order is slightly different. Easy shifters' standard deviation values for all ten factors are smaller than for the other two types. Difficult shifters score overall the lowest mean values across all three types of shifters but the highest values for standard deviation. Some significant differences between mean values were recorded in various combinations of two shifters types pairs (independent-samples $t$ test), but only 'reliable timetable' values were significantly different between all three types of shifters' pairs.

The values of the chi-squared test presented in Table 5 show that the results are significantly different within the three sub-samples statistically, but for some factors only. Within the staff sub-sample, the results are different between the shifters categories for six factors, compared with eight factors within the student sub-sample, where five factors appear for both sub-samples. The results for the 'lower price' factor are unique, as they are not significant within the first two groups of shifters but appear to be statistically significantly different when comparing the staff and students' results. These results show that price is not a factor that would differentiate results within the staff or within student samples when considered separately. However, when considered against each other, the results are indeed significantly different.

In addition, Table 6 offers more detailed results for the 'lower price' factor, revealing that (see the rows in bold) a cheaper price of the future metro line is seen as 'very important' by $51.6 \%$ of students in comparison with only $36.7 \%$ of staff. This is followed by 'important' views expressed by $31.3 \%$ of staff and $24.2 \%$ of students. 'Lower price' is seen as not important (or not at all important) by totals of only $10.3 \%$ of staff and $8.6 \%$ of students. Overall, the results show that the issue of the price of future metro 

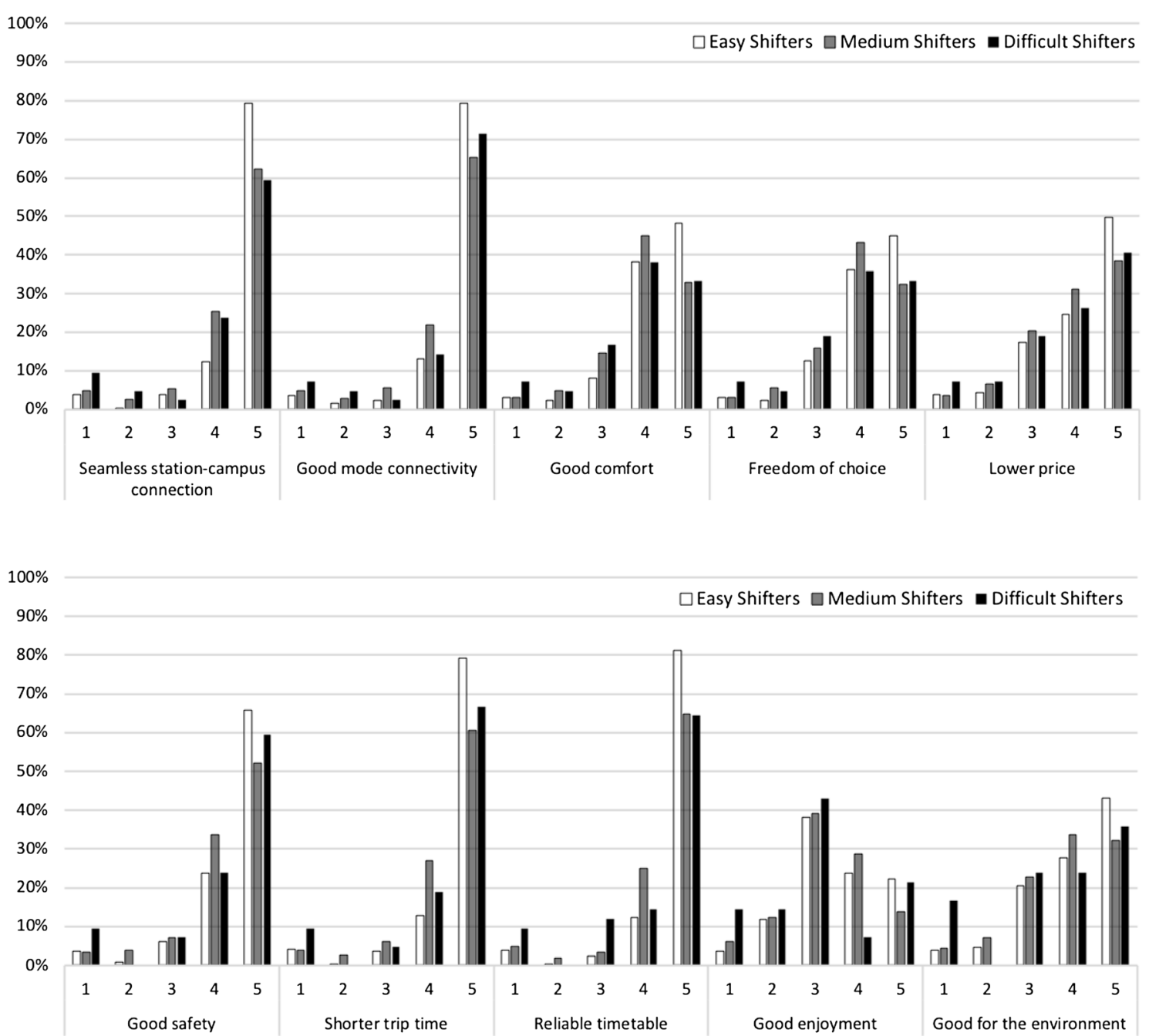

Fig. 5 Importance of factors that could facilitate respondents' shift to metro (\%)

Table 4 Mean values of 10 factors that could facilitate respondents' shift to metro with results for chi-squared test

\begin{tabular}{|c|c|c|c|c|c|c|c|c|c|c|c|}
\hline & \multicolumn{2}{|c|}{ Easy shifters } & \multicolumn{2}{|c|}{$\begin{array}{l}\text { Medium } \\
\text { shifters }\end{array}$} & \multicolumn{2}{|c|}{$\begin{array}{l}\text { Difficult } \\
\text { shifters }\end{array}$} & \multicolumn{2}{|l|}{ Total } & \multicolumn{3}{|l|}{ Chi-squared test } \\
\hline & Mean & SD & Mean & SD & Mean & SD & Mean & SD & Pearson chi-squared & df & Sig. \\
\hline Reliable timetable & 4.67 & 0.87 & 4.43 & 1.01 & 4.24 & 1.27 & 4.51 & 0.98 & 34.708 & 8 & 0.000 \\
\hline Good mode connectivity & $4.64 * *$ & 0.89 & 4.40 & 1.04 & 4.38 & 1.21 & 4.49 & 1.00 & 20.051 & 10 & 0.029 \\
\hline Seamless station-campus connection & $4.63 * *$ & 0.89 & 4.38 & 1.03 & 4.19 & 1.29 & 4.47 & 1.00 & 31.250 & 10 & 0.001 \\
\hline Shorter trip time & 4.62 & 0.92 & 4.38 & 0.98 & 4.33 & 1.22 & 4.47 & 0.98 & 32.829 & 8 & 0.000 \\
\hline Good safety & 4.48 & 0.92 & 4.27 & 0.98 & 4.24 & 1.23 & 4.35 & 0.98 & 21.699 & 8 & 0.006 \\
\hline Good comfort & $4.26 * *$ & 0.93 & 4.00 & 0.97 & 3.86 & 1.16 & 4.09 & 0.98 & 23.120 & 10 & 0.010 \\
\hline Freedom of choice & $4.19^{*}$ & 0.96 & 3.96 & 0.99 & 3.83 & 1.17 & 4.04 & 1.00 & 19.256 & 10 & 0.037 \\
\hline Lower price & $4.12 * *$ & 1.09 & 3.94 & 1.08 & 3.86 & 1.24 & 4.01 & 1.10 & - & - & - \\
\hline Good for the environment & $4.02 * *$ & 1.08 & 3.82 & 1.10 & 3.62 & 1.41 & 3.88 & 1.12 & 26.320 & 10 & 0.003 \\
\hline Good enjoyment & $3.50 * *$ & 1.07 & 3.32 & 1.05 & 3.07 & 1.30 & 3.37 & 1.08 & 24.085 & 10 & 0.007 \\
\hline$N$ & 260 & & 365 & & 42 & & 667 & & & & \\
\hline
\end{tabular}

$*_{n}=258 ; *^{*} n=259 ; \mathrm{SD}$, standard deviation 
Table 5 Statistically significant results of chi-squared tests for the 10 factors

\begin{tabular}{|c|c|c|c|c|c|c|c|c|c|}
\hline \multirow[t]{2}{*}{ Red Line use factor } & \multicolumn{3}{|l|}{ Staff metro shifters } & \multicolumn{3}{|l|}{ Student metro shifters } & \multicolumn{3}{|l|}{ Staff versus students } \\
\hline & $\begin{array}{l}\text { Pearson chi-squared } \\
\text { value }\end{array}$ & df & Sig. & $\begin{array}{l}\text { Pearson chi-squared } \\
\text { value }\end{array}$ & df & Sig. & $\begin{array}{l}\text { Pearson chi-squared } \\
\text { value }\end{array}$ & $\mathrm{df}$ & Sig. \\
\hline $\begin{array}{l}\text { Seamless station-campus } \\
\text { connection }\end{array}$ & 26.65 & 8 & 0.001 & 22.06 & 10 & 0.015 & - & - & - \\
\hline Good mode connectivity & 19.973 & 10 & 0.030 & 16.872 & 8 & 0.031 & - & - & - \\
\hline Good comfort & 19.44 & 10 & 0.035 & 19.185 & 8 & 0.014 & - & - & - \\
\hline Freedom of choice & - & - & - & 27.871 & 10 & 0.002 & - & - & - \\
\hline Lower price & - & - & - & - & - & - & 16.894 & 5 & 0.005 \\
\hline Good safety & 18.777 & 8 & 0.016 & - & - & - & - & - & - \\
\hline Shorter trip time & 27.425 & 8 & 0.001 & 20.309 & 8 & 0.009 & - & - & - \\
\hline Reliable timetable & 32.604 & 8 & 0.000 & 21.435 & 8 & 0.006 & - & - & - \\
\hline Good enjoyment & - & - & - & 24.757 & 8 & 0.002 & - & - & - \\
\hline Good for the environment & - & - & - & 17.322 & 8 & 0.027 & - & - & - \\
\hline
\end{tabular}

Table 6 Results for staff and students' responses to the 'lower price' factor

\begin{tabular}{|c|c|c|c|c|c|c|c|}
\hline & \multicolumn{6}{|c|}{ Red Line incentive: lower price than other options } & \multirow[t]{2}{*}{ Total } \\
\hline & 1-Not at all important & 2-Not important & 3-Neutral & 4-Important & 5-Very important & No response & \\
\hline \multicolumn{8}{|l|}{ Staff } \\
\hline Count & 14 & 26 & 84 & 122 & 143 & 1 & 390 \\
\hline Expected count & 15.2 & 22.2 & 74.3 & 110.5 & 167.2 & 0.6 & 390 \\
\hline$\%$ within staff or students & 3.6 & 6.7 & 21.5 & 31.3 & 36.7 & 0.3 & 100 \\
\hline$\%$ within lower price & 53.8 & 68.4 & 66.1 & 64.6 & 50.0 & 100.0 & 58.5 \\
\hline$\%$ of total & 2.1 & 3.9 & 12.6 & 18.3 & 21.4 & 0.1 & 58.5 \\
\hline Std. residual & -0.3 & 0.8 & 1.1 & 1.1 & -1.9 & 0.5 & \\
\hline \multicolumn{8}{|l|}{ Students } \\
\hline Count & 12 & 12 & 43 & 67 & 143 & 0 & 277 \\
\hline Expected count & 10.8 & 15.8 & 52.7 & 78.5 & 118.8 & 0.4 & 277 \\
\hline$\%$ within staff or students & 4.3 & 4.3 & 15.5 & 24.2 & 51.6 & 0.0 & 100 \\
\hline$\%$ within lower price & 46.2 & 31.6 & 33.9 & 35.4 & 50.0 & 0.0 & 41.5 \\
\hline$\%$ of total & 1.8 & 1.8 & 6.4 & 10.0 & 21.4 & 0.0 & 41.5 \\
\hline Std. residual & 0.4 & -1 & -1.3 & -1.3 & 2.2 & -0.6 & \\
\hline \multicolumn{8}{|l|}{ Total } \\
\hline Count & 26 & 38 & 127 & 189 & 286 & 1 & 667 \\
\hline Expected count & 26 & 38 & 127 & 189 & 286 & 1 & 667 \\
\hline$\%$ within staff or students & 3.9 & 5.7 & 19.0 & 28.3 & 42.9 & 0.1 & 100 \\
\hline$\%$ within lower price & 100 & 100 & 100 & 100 & 100 & 100 & 100 \\
\hline$\%$ of total & 3.9 & 5.7 & 19.0 & 28.3 & 42.9 & 0.1 & 100 \\
\hline
\end{tabular}

tickets is much more important to students than to staff, as one might expect.

\subsubsection{Metro Station Feeder Priorities}

Although in the Salaya case study the distance between the station and the campus is approx. $150 \mathrm{~m}$, the actual access is poor due to heavy traffic on the main road, the poor quality of a side road and an overall excess distance due to the location of internal campus walls and gates. It is recognised that access between the two locations will have to be improved if more staff, students and visitors are to be encouraged to shift to the metro mode in the future. In terms of priorities, when choosing a future station-campus 


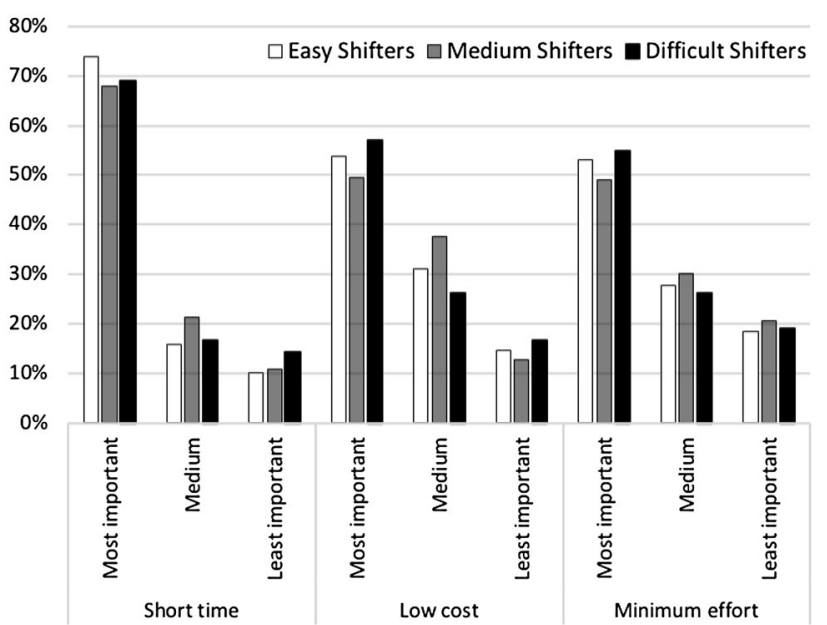

Fig. 6 Respondents' station-campus feeder priorities (\%)

feeder option, respondents were asked to put three parameters (short time, low cost and minimum effort) in order from 1-'most important', 2-'medium' to 3-'least important'. Figure 6 and Table 7 display these results divided by the three shifters categories. Overall, the trends for each parameter are similar between the shifters, with 'short time' being the most important parameter for all shifters (between 67\% and 73\%). The second priority is 'low cost', and the third is 'minimum effort', but the overall difference between them is small, with the two parameters being slightly more important for difficult shifters than easy or medium shifters. This suggests that, when considering a new station-campus feeder option, the priority should be short time first, followed by (nearly) equal weight put on both cost and effort.

\section{Conclusions and Recommendations}

This paper focusses on commuters' willingness to shift to metro and conditions under which such a shift would be considered. A case study of a new metro line linking Salaya and Bangkok, Thailand, was investigated. A sample of 667 respondents, composed of staff and students based at a Salaya university campus, who are potential users of the new metro line, was collected. Of the sample, $90 \%$ declared a willingness to shift to metro, and they were further split into easy (use metro every time) and medium (use metro sometimes) shifters. The results revealed that, firstly, younger respondents are more keen to shift while, the older the respondents, the greater the proportion of difficult shifters within their age categories. This shows that older respondents have clearer opinions for or against the shift in comparison with the younger sample.

Secondly, within each type of shifters, $64 \%$ or more of respondents already use public transport for their SalayaBangkok commute. This presents a great opportunity for the new metro operator to attract existing public transport users and come up with strategies to facilitate their shift to metro. Also, there is a small market for private transport users to shift to metro, but marketing strategies for them would have to be specifically tailored to clearly highlight benefits (e.g. environmental) of metro over a car or a motorcycle option.

Finally, the top four factors that would help to facilitate respondents' shift to metro in the future are: 'reliable timetable', 'good mode connectivity', 'seamless stationcampus connection' and 'shorter trip time'. These results show that service reliability, connectivity and time factors are crucial for the respondents in choosing metro over other modes in their future commuting trips between Salaya and Bangkok. Therefore, it is recommended that the future metro operator focus on these four factors when designing the service. In order to achieve this, in addition to excellent operations, effective collaboration between metro stakeholders will be required to ensure that access to stations is seamless (with time, cost and effort as priorities for feeder options) and links with other transport modes are well facilitated. Moreover, there are differences between staff and students in the way they perceive ticket price as a factor which could motivate them to shift, where the future metro ticket price is 'very important' to half of the students but to only a third of the staff in the sample. This suggests that different strategies would be advisable to attract different types of customers to use metro in the future.

\section{Limitations and Next Steps}

Three key limitations of the study can be identified as follows: Firstly, due to delays related to internal survey approval procedures, the data collection took place in May and June, which is the end of the academic year in
Table 7 Mean values of respondents' station-campus feeder priorities

\begin{tabular}{|c|c|c|c|c|c|c|c|c|c|c|c|c|}
\hline & \multicolumn{3}{|c|}{ Easy shifters } & \multicolumn{3}{|c|}{ Medium shifters } & \multicolumn{3}{|c|}{ Difficult shifters } & \multicolumn{3}{|l|}{ Total } \\
\hline & Mean & $n$ & SD & Mean & $n$ & SD & Mean & $n$ & SD & Mean & $n$ & SD \\
\hline Short time & 1.36 & 259 & 0.658 & 1.43 & 365 & 0.678 & 1.45 & 42 & 0.739 & 1.40 & 666 & 0.674 \\
\hline Low cost & 1.61 & 259 & 0.731 & 1.63 & 364 & 0.698 & 1.60 & 42 & 0.767 & 1.62 & 665 & 0.715 \\
\hline Minimum effort & 1.65 & 258 & 0.776 & 1.71 & 364 & 0.786 & 1.64 & 42 & 0.791 & 1.69 & 664 & 0.782 \\
\hline
\end{tabular}


Thailand, with student exams and holidays. This could have had an effect on the quality and quantity of data collected and the fact that the final sample is dominated by staff. If a similar study were to be repeated with a Thai university sample, a more convenient time period, possibly between September and November, would be recommended for data collection.

Secondly, a survey is a tool that allows for collection of a large sample and offers a quantitative approach to the topic, but limits the interaction between researchers and respondents. It would have been beneficial to understand respondents' motivations behind their answers to some of the questions, and these were not explored further in the survey. A follow-up in-depth interview with respondents would allow for a more quantitative approach to the study and for better understanding of respondents' motivations behind supporting or rejecting their potential shift to metro in the future.

Finally, a university-based sample represents a specific community with its unique commute patterns and socioeconomics, which do not necessarily represent the wider population [34]. Therefore, it must be highlighted that the results of the study are not representative of the general population located along the new metro lines, but are specific to this academic population located at a Salaya university campus. Further study at other locations across the new metro network, including large employers and neighbourhood communities, would identify whether results presented in the paper are Salaya specific only or are more general outcomes applicable to both university- and non-university-based samples.

Acknowledgements This work was supported by the research postdoctoral sponsorship of Mahidol University awarded to Dr. Anna Fraszczyk in 2016.

Open Access This article is distributed under the terms of the Creative Commons Attribution 4.0 International License (http://crea tivecommons.org/licenses/by/4.0/), which permits unrestricted use, distribution, and reproduction in any medium, provided you give appropriate credit to the original author(s) and the source, provide a link to the Creative Commons license, and indicate if changes were made.

\section{References}

1. UITP (2018) Statistics brief. World Metro Figures 2018. Available at: https://www.uitp.org/sites/default/files/cck-focus-papersfiles/Statistics\%20Brief\%20-\%20World\%20metro\%20figures\% 202018V4_WEB.pdf. Accessed 9 June 2019

2. Fraszczyk A, Mulley C (2016) Public perception of and attitude to driverless train: a case study of Sydney, Australia. Urb Rail Transit 3(2):100-111

3. Dahlan AF (2019) A comparative study of various transport modes in Jakarta in the light of a new MRT service. Master Thesis. Mahidol University, Salaya
4. De Witte A, Hollevoet J, Dobruszkes F, Hubert M (2013) Linking modal choice to motility: a comprehensive review. Transp Res Part A 49(2013):329-341

5. Derek Halden Consultancy (2003) Barriers to modal shift. Report. Available at https://www.webarchive.org.uk/wayback/ archive/20180520122845/http://www.gov.scot/Publications/ 2003/09/18178/26386 Accessed 2 Aug 2019

6. UN (2019) The Sustainable Development Goals Report 2019

7. Caliper (2019) Caliper mapping and transportation glossary. Available at https://www.caliper.com/glossary/what-are-modechoice-models.htm Accessed 16 Sept 2019

8. Ratrout NT, Gazder U, Al-Madani HMN (2014) A review of mode choice modelling techniques for intra-city and border transport. World Rev Intermodal Transp Res 5(1):2014

9. Mitsubishi Research Institute (2011) New mechanism feasibility study for development of mass rapid transit (MRT) systems in Jakarta, Indonesia, and Hanoi and Ho Chi Minh, Viet Nam. New Mechanism Feasibility Study 2011-Final Report. Available at https://www.scribd.com/document/236688541/MRT-FeasibilityStudy-JAKARTA. Accessed 13 June 2019

10. NECA and Nexus (2016) Metro and Local Rail Strategy. Available at https://www.nexus.org.uk/sites/default/files/Metro\% 20and\%20Light\%20Rail\%20Strategy\%20Draft_0.pdf. Accessed 13 June 2019

11. Otsu H (2008) Thailand MRTA initial system project (Blue Line) I-V. https://www.jica.go.jp/english/our_work/evaluation/oda_ loan/post/2008/pdf/e_project09_full.pdf. Accessed 13 June 2019

12. AECOM (2018) Crenshaw northern extension feasibility/alternatives analysis study. Final Report. https://media.metro.net/pro jects_studies/crenshaw_northern_extension/images/feasibility_ report_final_crenshaw_north.pdf. Accessed 13 June 2019

13. Sydney Metro (2018) Sydney metro northwest interchange access plan. https://www.sydneymetro.info/sites/default/files/documentlibrary/Sydney_Metro_Northwest_Interchange_Access_plan.pdf. Accessed 15 June 2019

14. Sydney Metro (2017) The Sydney metro sustainability report 2017. https://www.sydneymetro.info/sites/default/files/documentlibrary/SydneyMetro_SustainabilityReport2017.pdf. Accessed 15 June 2019

15. Transport Focus (2018) National Rail Passenger Survey: Autumn 2018-Main report. https://www.transportfocus.org.uk/researchpublications/publications/national-rail-passenger-survey-nrpsautumn-2018-main-report/. Accessed 15 June 2019

16. Flyvbjerg B, Bruzelius N, Rothengatter W (2007) Megaprojects and risk: an anatomy of ambition. Cambridge University Press, Cambridge

17. Flyvbjerg B (2005) Measuring inaccuracy in travel demand forecasting: methodological considerations regarding ramp up and sampling. Transp Res A Pol Pract 39(6):522-530

18. Flyvbjerg (2007) Truth and lies about megaprojects. Inaugural speech. www.researchgate.net. Accessed 1 Aug 2019

19. JICA (2002) Calcutta Metro railways (Phase II) construction project. https://www.jica.go.jp/english/our_work/evaluation/oda_ loan/post/2002/pdf/109_full.pdf. Accessed 1 Aug 2019

20. Bangkok Post (2016) Fare cut boosts Purple Line riders, but still below target. https://property.bangkokpost.com/news/1081544/ fare-cut-boosts-purple-line-riders-but-still-below-target. Accessed 15 June 2019

21. Weerawat W, Thongboonpian T, Fraszczyk A (2019) Impact of a "Missing Link" on passenger's travel time: a case study of Tao Poon Station. In: Marinov M, Fraszczyk A (eds) Sustainable rail transport. Lecture notes in mobility. Springer, Cham

22. Bangkok Post (2017) Purple Line use up 47\% since 'missing link' completed. https://www.bangkokpost.com/news/transport/ 1320863/purple-line-use-up-47-since-missing-link-completed. Accessed 15 June 2019 
23. The Nation (2018) Bangkok's planned rail system expected to be on par with London's by 2025. http://www.nationmultimedia. com/detail/Real_Estate/30346016. Accessed 15 June 2019

24. O'Sullivan M (2019) Glitches fail to deter commuters as 546,000 people board metro trains. The Sydney Morning Herald. 2 June 2019. https://www.smh.com.au/national/nsw/glitches-fail-todeter-commuters-as-546-000-people-board-metro-trains20190528-p51rua.html. Accessed 15 June 2019

25. Sydney Metro (2019) About Sydney metro. https://www.sydney metro.info/about. Accessed 15 June 2019

26. Sohoni AV, Thomas M, Krishna Rao KV (2016) Mode shift behavior of commuters due to the introduction of new rail transit mode. Transp Res Proc 25(2017):2603-2618

27. MRTA (2016) Mass rapid transit authority of Thailand. Presentation by MRT project development department. https://www. unescap.org/sites/default/files/MRTA\%20Thailand.pdf. Accessed 25 July 2018

28. Siemens (2018) A Master Plan to Counteract non-stop Traffic Congestion. pictures-of-the-future/mobility-andmotors/urbanmobility-publictransport-in-bangkok.html. Accessed 25 July 2018

29. Charanwanitwong T, Fraszczyk A (2018) Rail liberalisation in Europe and lessons for Thailand: Policy makers vs. academic views. Transp Res Part A 113(2018):421-440

30. University of Tasmania (2015) 2015 Travel behaviour survey: summary of findings. December 2015

31. ITS UC Davies (2016) Results of the 2015-2016 Campus Travel Survey. September 2016

32. University of Bristol (2014) University of Bristol Combined Travel Plan 2009-2016

33. TPS (2015) Northumbria University: Student and Staff Travel Survey 2014. Report on Findings. May 2015

34. Khattak A, Wang X, Son S et al (2011) Travel by university students in Virginia: Is this travel different from travel by the general population? Transp Res Rec J Transp Res Board 2011:137-145 